\title{
The Role of the Transcription Factor SIM2 in Prostate Cancer
}

\author{
Bin Lu ${ }^{1}$, John M. Asara ${ }^{2}$, Martin G. Sanda ${ }^{1 *}$, Mohamed S. Arredouani ${ }^{1 *}$
}

1 Department of Surgery, Beth Israel Deaconess Medical Center, Harvard Medical School, Boston, Massachusetts, United States of America, 2 Department of Medicine, Beth Israel Deaconess Medical Center, Harvard Medical School, Boston, Massachusetts, United States of America

\begin{abstract}
Background: Recent reports have suggested a possible involvement of Single-minded homolog 2 (SIM2) in human solid cancers, including prostate cancer. However, the exact role of SIM2 in cancer in general, and in prostate cancer in particular, remains largely unknown. This study was designed to elucidate the role of SIM2 in prostate cancer using a shRNA-based approach in the PC3 prostate cancer cell line.
\end{abstract}

Methods: Lentiviral shRNAs were used to inhibit SIM2 gene and protein levels in PC3 cells. Quantitative RT-PCR and branched DNA were performed to evaluate transcript expression. SIM2 protein expression level was measured by western blot. Profiling of gene expression spanning the whole genome, as well as polar metabolomics of several major metabolic pathways was performed to identify major pathway dysregulations.

Results: SIM2 gene and protein products were significantly downregulated by lenti-shRNA in PC3 cell line. This low expression of SIM2 affected gene expression profile, revealing significant changes in major signaling pathways, networks and functions. In addition, major metabolic pathways were affected.

Conclusion: Taken together, our results suggest an involvement of SIM2 in key traits of prostate tumor cell biology and might underlie a contribution of this transcription factor to prostate cancer onset and progression.

Citation: Lu B, Asara JM, Sanda MG, Arredouani MS (2011) The Role of the Transcription Factor SIM2 in Prostate Cancer. PLoS ONE 6(12): e28837. doi:10.1371/ journal.pone.0028837

Editor: Klaus Roemer, University of Saarland Medical School, Germany

Received August 26, 2011; Accepted November 16, 2011; Published December 9, 2011

Copyright: ( 2011 Lu et al. This is an open-access article distributed under the terms of the Creative Commons Attribution License, which permits unrestricted use, distribution, and reproduction in any medium, provided the original author and source are credited.

Funding: This work is supported by NIH-NCI Early Detection Research Network grant UO1-CA11391 (M. Sanda), Department of Defense Prostate Cancer Training Award W81XWH-09-1-0626 (B. Lu), and Prostate Cancer Foundation Young Investigator Award (M.S. Arredouani). The funders had no role in study design, data collection and analysis, decision to publish, or preparation of the manuscript.

Competing Interests: The authors have declared that no competing interests exist.

* E-mail: marredou@bidmc.harvard.edu (MSA); msanda@bidmc.harvard.edu (MGS)

\section{Introduction}

Single-minded homolog 2 (SIM2) gene is located on the human chromosome 21q22.2 and is a member of the basic helix-loop-helix PAS [per-Arnt-Sim] (bHLH-PAS) family of transcription factors $[1,2]$. SIM2 was originally thought to contribute to Down's syndrome (DS) [3]. As a transcription factor (TF), murine SIM2 (mSIM2) mediates gene expression through GNS midline enhancer (CME) element with its dimerization partner ARNT via ARNT carboxy-terminus [4]. The transcription factor c-myb regulates SIM2 transcription in glioblastoma cells, and a nuclear localization signal (NLS) mediates nuclear localization of SIM2 [5].

A prior in silico bioinformatics approach using the Cancer Genome Anatomy Project (CGAP) database of the National Cancer Institute (NCI) identified SIM2 as associated with colon, pancreas and prostate carcinomas, while absent in the corresponding normal tissues [6]. Two different spliced isoforms of SIM2 transcript, SIM2long (SIM2-l) and SIM2-short (SIM2-s), have been reported while their differential function in humans are not known yet [1]. SIM2-s was specifically expressed in early stages of colon cancer. Antisense inhibition of SIM2-s expression by antisense oligos caused growth inhibition and apoptosis in colon cancer cell line RKO and tumor growth in nude mice and also in pancreatic cancer cell line CAPAN-
$1[7,8]$. Apoptosis was induced by SIM2-s inhibition in the RKO colon cancer cell line [9]. SIM2-s was also found to have tumor suppressive activity in breast cancer [10]. The invasion potential of glioblastoma was decreased significantly by SIM2s inhibition, consistent with a decrease in the expression of matrix metalloproteinase 2 at both mRNA and protein levels [11].

We have previously reported SIM2 as a potential biomarker and immunotherapy target for human prostate cancer [12]. Although SIM2-s expression (as measured by immunohistochemistry of prostatectomy specimens) has been associated with aggressive histopathology in prostate cancer, and overexpressing ectopic SIM2s enhanced survival in certain conditions in PC3AR+ cells [13,14], the functional role of SIM2 gene in prostate cancer cell is largely unknown.

In this study we sought to elucidate the functional role of SIM2 in PCa using a gene silencing approach and characterization of molecular and functional changes by both gene expression profiling and metabolomic profiling.

\section{Materials and Methods}

\section{Cell lines}

The human PC3, LNCaP, VCaP and DU145 cell lines were purchased from the American Type Culture Collection (ATCC, 
Manassas, VA) and cultured as per ATCG's protocol. Benign PrEG cells, as described in Berger $\mathrm{R}$ et al, 2004, were kindly provided by Dr. W. Hahn at Dana-Farber Cancer Institute, Boston, MA.

\section{Transduction Particles}

The pLKO.1-puro control lentiviral transduction particles, MISSION luciferase shRNA control lentiviral transduction particles and MISSION SIM2 shRNA lentiviral transduction particles were used to infect PC3 cell line (Sigma-Aldrich, Saint Louis, MO).

\section{Sample selection, RNA purification and reverse transcription}

Ten benign and fourteen tumor radical prostatectomy tissue samples were obtained and total RNAs were processed as described in our previous work [12]. Cell line total RNA was isolated using TRIzol reagent (Invitrogen Corporation, Carlsbad, CA) according to the manufacturer's instructions. Purified RNA was quantified by NanoDrop ND-1000 Spectrophotometer (NanoDrop, Wilmington, DE). $500 \mathrm{ng}$ of each cell total RNA was reverse transcribed into cDNA using oligo dT and superscript III reverse transcriptase (Invitrogen Corporation, Carlsbad, CA) under the manufacturer's instructions.

\section{Gene expression microarrays and analysis}

$250 \mathrm{ng}$ total RNA was amplified using Ambion's MessageAmp II mRNA Amplification kit. Biotin-UTP was incorporated during the overnight in vitro transcription step according to the manufacturer's protocol. Gene expression was assessed using Affymetrix's (Santa Clara, CA) GeneChip U133 array (Plus 2.0 chip) arrays representing the whole human genome transcripts. $15 \mu \mathrm{g}$ cRNA was fragmented and hybridized to arrays' according to the manufacturer's protocols as described previously [15]. The quality of scanned arrays images were determined on the basis of background values, percent present calls, scaling factors, and $3^{\prime}-5^{\prime}$ ratio of $\beta$-actin and GAPDH using the BioConductor $\mathrm{R}$ packages. The signal value for each transcript was summarized using PM-only based signal modeling algorithm described in dchip. The PM only based modeling based algorithm yields less number of false positives as compared to the PM-MM model. In this way, the signal value corresponds to the absolute level of expression of a transcript [16]. These normalized and modeled signal values for each transcript were used for further high level bioinformatics analysis. During the calculation of model based expression signal values, array and probe outliers are interrogated and images spike are treated as signal outliers. The outlier detection was carried out using dchip outlier detection algorithm. A chip is considered as an outlier if the probe, single or array outlier percentage exceeds a default threshold of $5 \%$. When comparing two groups of samples to identify genes enriched in a given phenotype, if $90 \%$ lower confidence bound (LCB) of the fold change $(\mathrm{FC}$ ) between the two groups was above 1.2, the corresponding gene was considered to be differentially expressed. $\mathrm{LCB}$ is a stringent estimate of $\mathrm{FC}$ and has been shown to be the better ranking statistic [17] It has been suggested that a criterion of selecting genes that have a LCB above 1.2 most likely corresponds to genes with an "actual" fold change of at least 2 in gene expression [18]. Data were extracted from CEL files and normalized using RMAexpress (http://rmaexpress.bmbolstad.com/). Data were analyzed using $\mathrm{MeV}$ software (http://www.tm4.org/mev/).

\section{Cell signaling pathway analysis}

The Ingenuity Pathways Analysis (Ingenuity Systems ${ }^{\circledR}$, http:// www.ingenuity.com) applications were used to generate networks and assess statistically relevant biofunctions, canonical pathways and networks associated with the differentially expressed gene profiles extracted from the transcriptome data.

Branched DNA and quantitative Real-Time PCR (qRT-PCR)

Branched DNA was performed to evaluate the SIM2s and SIM2L gene expression in the human prostate total RNA samples and normalized by 2 control genes ALSAl and HPRT (QuantiGene 2.0 Reagent System, Affymetrix Inc, Fremont, CA). For quantitative RT-PCR, $1 \mu \mathrm{l}$ cDNA was used for each well RT-PCR reactions. Samples were performed in triplicates. Taqman universal PCR Master Mix (Applied Biosystems, Foster City, CA) was used for two-step real-time RT-PCR analysis on Applied Biosystems 7900HT Prism instrument. Taqman real time PGR primers for GAPDH (4310884E) and SIM2L (hs00231925_ml) were purchased from Applied Biosystems (Foster Gity, CA). Taqman real time PGR primers for SIM2s were designed by our group and purchased from Biosearch Technologies (Novato, CA). SIM2s forward primer: 5'-gtgccaagct acgaaggtg-3'; SIM2s reverse primer: 5'-acttagaagcagaaagagggcaag-3'; probe: TCAGGTCTGGTCGTGGGGAAGGTG. Expression value of SIM2s or SIM2L in a given sample was normalized to the corresponding expression of GAPDH. The $2^{-\Delta \Delta \mathrm{Ct}}$ method was used to calculate relative expression of SIM2 gene as described previously $[19,20]$.

\section{Lentiviral transduction and stable cell line selection}

1.6 X $10^{4}$ PC3 cells were plated in 96 well plate and incubated for 20 hours. Medium was removed and $110 \mathrm{ul}$ of fresh medium containing hexadimethrine bromide to a final concentration of 8 $\mathrm{ug} / \mathrm{ml}$ were added. Lentiviral particles were added to appropriate wells at 5 MOI (multiplicity of Infection) and incubated overnight. Fresh medium was then added and cells cultured for 2 days, followed by a $10-12$ days culture with puromycin $(2 \mathrm{ng} / \mathrm{ml})$ added every 3 days.

Transient transduction was achieved over a 3-day incubation.

\section{Western blot}

Cells were washed twice with PBS twice before they were harvested by scraping. Cell lysates were prepared in cell lysis buffer (50 mmol/L Tris-HCl pH 8.0, $20 \mathrm{mM} \mathrm{EDTA,} \mathrm{1 \%} \mathrm{SDS,} \mathrm{and} 100$ $\mathrm{mM} \mathrm{NaCl}$ ) containing an enzyme inhibitor mixture tablet (Roche Molecular Biochemicals, Indianapolis, IN) and PMSF (SigmaAldrich, Saint Louis, MO). Protein concentration was determined using BCA protein assay kit (Thermo Scientific, Rockford, IL). A total of $20-50 \mu \mathrm{g}$ of protein extract was fractionated by SDSPAGE and transferred to a polyvinylidene difluoride membrane (Immobilon-P; Millipore). The membrane was blocked with TBS$\mathrm{T}(0.1 \%$ Tween 20 in PBS) containing 3\% dry milk and incubated with SIM2s primary antibody (Santa Cruz, sc-8715, isoform NM_009586) overnight at $4^{\circ} \mathrm{C}$. After three washes with TBS-T, the membrane was incubated with HRP-conjugated secondary Ab for $1 \mathrm{~h}$ and then washed with $0.05 \%$ Tween 20 in PBS. The immune complexes were detected by ECL methods (Thermo Scientific, Rockford, IL).

\section{Metabolite profiling using Targeted Liquid- Chromatography Tandem Mass Spectrometry (LC/MS/ MS)}

$10^{6}$ cells exponentially growing in basal media with dialyzed serum were harvested in $3 \mathrm{~mL} 80 \% \mathrm{v} / \mathrm{v}$ HPLC grade methanol at dry ice temperatures. Fresh media was added 24 hours and 2 hours prior to the extraction. Insoluble material in lysates was 
Table 1. Clinical information and SIM2 gene expression of 10 normal and 14 tumor prostatectomy evaluated by branched DNA technique.*

\begin{tabular}{|c|c|c|c|c|c|c|}
\hline Case number & $\begin{array}{l}\text { Tumor }=1 \\
\text { normal }=0\end{array}$ & $\begin{array}{l}\text { Gleason } \\
\text { score }\end{array}$ & $\begin{array}{l}\text { PSA level } \\
\text { (ng/ml) }\end{array}$ & $\begin{array}{l}\text { Sim2s mRNA } \\
\text { expression ** }\end{array}$ & $\begin{array}{l}\text { Sim2L mRNA } \\
\text { expression ** }\end{array}$ & Sim2s/Sim2L ratio \\
\hline 315 & 0 & $\mathrm{~N} / \mathrm{A}$ & 7.6 & 0.0025 & 0.0024 & 1.041667 \\
\hline 318 & 0 & $\mathrm{~N} / \mathrm{A}$ & 8.4 & 0.0057 & 0.008 & 0.7125 \\
\hline 322 & 0 & $\mathrm{~N} / \mathrm{A}$ & 6.5 & 0.0182 & 0.0197 & 0.923858 \\
\hline 334 & 0 & $\mathrm{~N} / \mathrm{A}$ & 5 & 0.0368 & 0.0645 & 0.570543 \\
\hline 91 & 0 & $\mathrm{~N} / \mathrm{A}$ & 5 & 0.0215 & 0.0321 & 0.669782 \\
\hline 20 & 0 & $\mathrm{~N} / \mathrm{A}$ & 7.2 & 0.01 & 0.0233 & 0.429185 \\
\hline 149 & 0 & $\mathrm{~N} / \mathrm{A}$ & 7.1 & 0.0045 & 0.0057 & 0.789474 \\
\hline 516 & 0 & $\mathrm{~N} / \mathrm{A}$ & 13.5 & 0.029 & 0.0379 & 0.765172 \\
\hline 524 & 0 & $\mathrm{~N} / \mathrm{A}$ & 11.5 & 0.0338 & 0.0805 & 0.419876 \\
\hline 544 & 0 & $\mathrm{~N} / \mathrm{A}$ & 7.8 & 0.0241 & 0.1094 & 0.220293 \\
\hline 411 & 1 & $3+4=7$ & 0.4 & 0.1449 & 0.2734 & 0.529993 \\
\hline 417 & 1 & $3+4=7$ & 4.2 & 0.2041 & 0.2281 & 0.894783 \\
\hline 471 & 1 & $3+4=7$ & 26.7 & 0.0414 & 0.0375 & 1.104 \\
\hline 474 & 1 & $3+3=6$ & 5.2 & 0.1711 & 0.2817 & 0.607384 \\
\hline 478 & 1 & $3+4=7$ & 4.4 & 0.0543 & 0.0921 & 0.589577 \\
\hline 482 & 1 & $3+4=7$ & 8.3 & 0.0513 & 0.1066 & 0.481238 \\
\hline 523 & 1 & $3+4=7$ & 8.7 & 0.2476 & 0.3987 & 0.621018 \\
\hline 539 & 1 & $3+3=6$ & 0.7 & 0.1956 & 0.5371 & 0.364178 \\
\hline 545 & 1 & $3+3=6$ & 3 & 0.1005 & 0.1996 & 0.503507 \\
\hline 547 & 1 & $3+3=6$ & 0.4 & 0.0824 & 0.134 & 0.614925 \\
\hline 548 & 1 & $4+3=7$ & 5.8 & 0.0606 & 0.1403 & 0.431932 \\
\hline 303 & 1 & $\mathrm{~N} / \mathrm{A}$ & 6.4 & 0.0631 & 0.1084 & 0.582103 \\
\hline 14 & 1 & $\mathrm{~N} / \mathrm{A}$ & 1.6 & 0.1812 & 0.2005 & 0.903741 \\
\hline 125 & 1 & $4+3=7$ & 18 & 0.0333 & 0.0494 & 0.674089 \\
\hline
\end{tabular}

*Prostatectomy samples were from Hershey Tissue Bank at Beth Israel Deaconess Medical Center, Boston.

**mRNA expression values were normalized by ALSA1 and HPRT genes by branched DNA technique.

doi:10.1371/journal.pone.0028837.t001
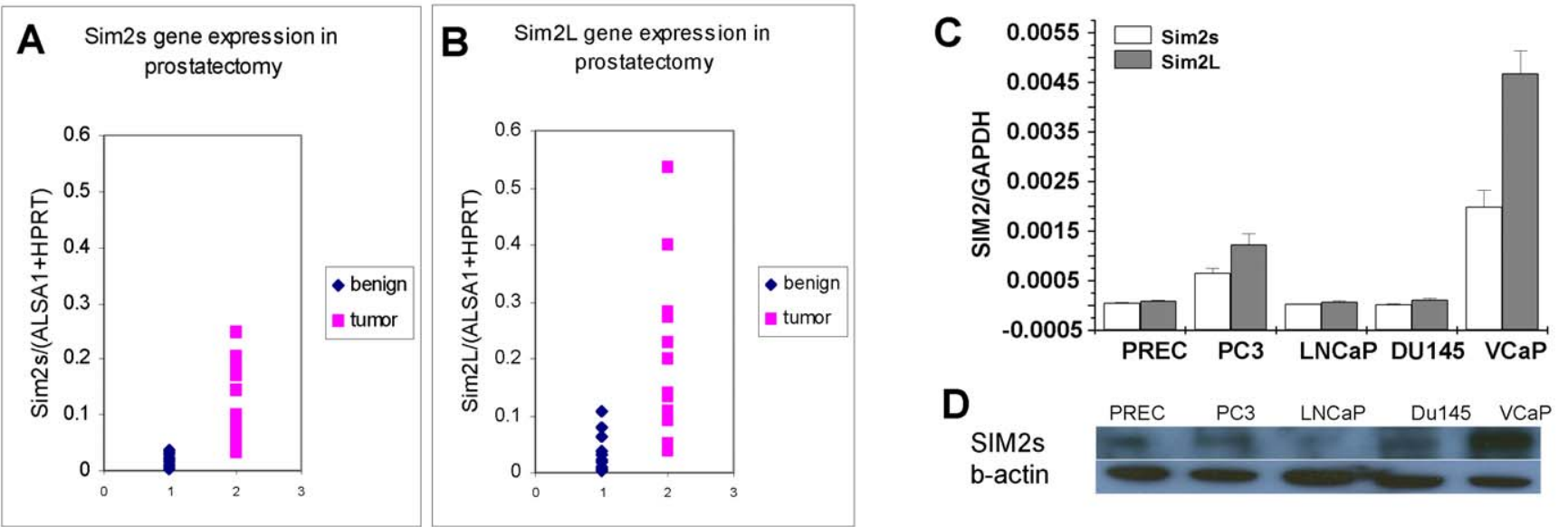

Figure 1. SIM2 expression in human prostatectomy, prostate normal and cancer cell lines. Quantitative Expression of SIM2 short isoform (A) and SIM2 long isoform (B) were evaluated by branched DNA technique in 10 normal and 14 human cancer prostatectomy specimens. Data were quantified using ALSA1 and HPRT as the normalizers. (C) Quantification of SIM2 short and long isoforms' expression in human prostate normal and cancer cell lines by real time RT-PCR. Data were quantified by the $\Delta \Delta \mathrm{C}_{\mathrm{T}}$ method and normalized to GAPDH. Column in white represents SIM2 short isoform and column in gray represents SIM2 long isoform. (D) Western blot were performed in prostate normal and cancer cell lines for SIM2s. doi:10.1371/journal.pone.0028837.g001 


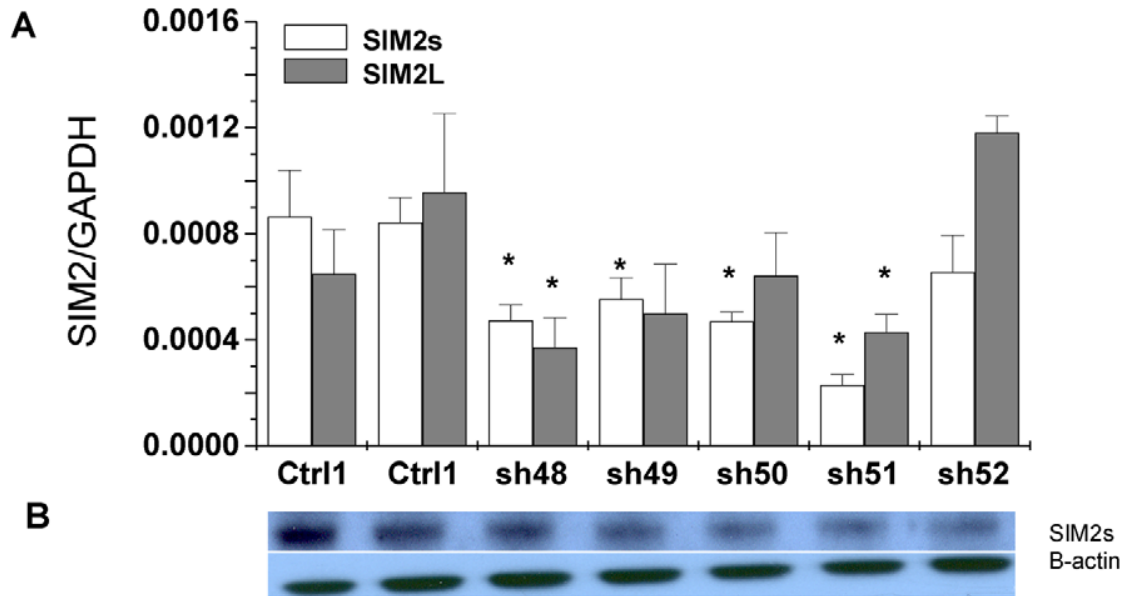

Figure 2. SIM2 expression in PC3 cells is downregulated by shRNA. Real time RT-PCR was performed in triplicates (A) and protein expression was evaluated by western blot (B). Control 1: Luciferase shRNA vector, Control 2: PLKO vector. sh48, sh49, sh50, sh50, sh51 and sh52: vectors expressing shRNAs targeting SIM2 gene at different sites. Column with "**" represents significant downregulation of SIM2 gene expression by shRNA comparing to both of control 1 and control $2(\mathrm{P}<0.05)$.

doi:10.1371/journal.pone.0028837.g002

centrifuged at 4000 RPM for 15 minutes and the resulting supernatant (metabolite content) was evaporated using a refrigerated SpeedVac to a pellet. Samples were re-suspended using $20 \mu \mathrm{L}$ HPLC grade water for mass spectrometry analysis. $10 \mu \mathrm{L}$ were injected and analyzed using a 5500 QTRAP triple quadrupole mass spectrometer (AB/Sciex) coupled to a Prominence UFLC HPLC system (Shimadzu ) via selected reaction monitoring (SRM) of a total of 255 endogenous water soluble metabolites for steady-
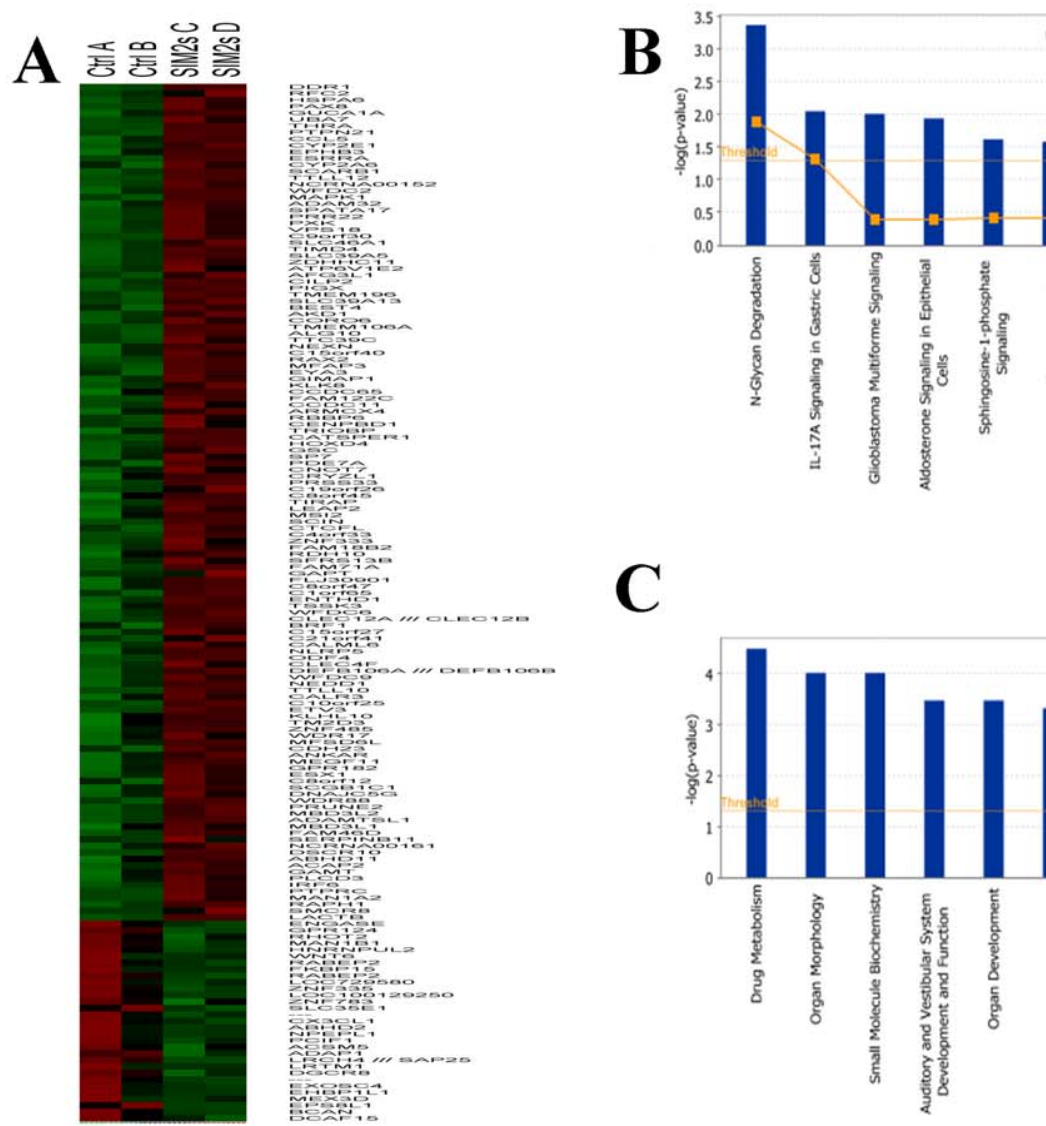

\section{Canonical Pathways}
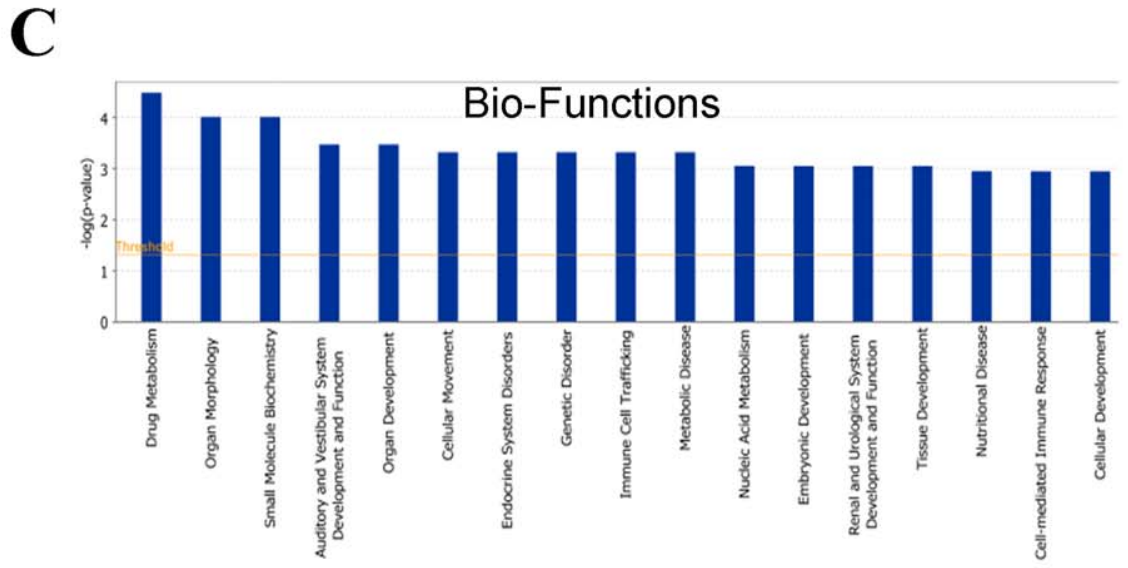

Figure 3. Heat map and cell signaling analysis for the dysregulated genes in SIM2 ${ }^{\text {low }}$ comparing to control PC3 cells. A. Control A: PC3 luciferase shRNA; Control B: PC3 PLKO vector; SIM2 C: SIM2 sh48; SIM2 D: SIM2 sh51. Gene expressions were either up or down greater than 2 fold in the SIM2 ${ }^{\text {low }}$ were listed. B. Top dysregulated signaling pathways in SIM2 ${ }^{\text {low }}$ PC3 cells. C. Top dysregulated cell functions in SIM2 ${ }^{\text {low }}$ PC3 cells. doi:10.1371/journal.pone.0028837.g003 
state analyses of samples. Some metabolites were targeted in both positive and negative ion mode for a total of 298 SRM transitions. ESI voltage was $+4900 \mathrm{~V}$ in positive ion mode and $-4500 \mathrm{~V}$ in negative ion mode. The dwell time was $5 \mathrm{~ms}$ per SRM transition and the total cycle time was 2.09 seconds. Approximately 8-10 data points were acquired per detected metabolite. Samples were delivered to the MS via normal phase chromatography using a 2.0 mm i.d x $15 \mathrm{~cm}$ Luna NH2 HILIC column (Phenomenex) at 285 $\mu \mathrm{L} / \mathrm{min}$. Gradients were run starting from $85 \%$ buffer B (HPLC grade acetonitrile) to $42 \% \mathrm{~B}$ from $0-5$ minutes; $42 \% \mathrm{~B}$ to $0 \% \mathrm{~B}$ from 5-16 minutes; $0 \% \mathrm{~B}$ was held from $16-24$ minutes; $0 \% \mathrm{~B}$ to $85 \%$ B from 24-25 minutes; $85 \%$ B was held for 7 minutes to reequilibrate the column. Buffer A was comprised of $20 \mathrm{mM}$ ammonium hydroxide $/ 20 \mathrm{mM}$ ammonium acetate $(\mathrm{pH}=9.0)$ in 95:5 water:acetonitrile. Peak areas from the total ion current for each metabolite SRM transition were integrated using MultiQuant vl.1 software (AB/Sciex).

Measurements were performed in triplicates and data were normalized per cell number. Only metabolites that were determined in all 6 samples were kept and analyzed using MetaboAnalyst [21,22].

\section{Statistical analysis}

Gene expression array data were analyzed as described under Materials and Methods. Based upon our earlier work [12], we tested for SIM2 upregulation in tumors versus controls with a one-sided t-test and compared against a p-value threshold of 0.05 .

Quantitative Real-Time PGR (qRT-PGR). Validation of differentially expressed genes was performed by qRT-PCR. $200 \mathrm{ng}$ of high quality RNA samples were reverse transcribed to first strand cDNA and $1 \mu \mathrm{l}$ CDNA was used for each well RT-PCR reaction. Samples were performed in triplicates. SYBR Green PCR Master Mix (Applied Biosystems, Foster City, CA) was used for two-step real-time RT-PCR analysis on Applied Biosystems 7900 HT Prism instrument. PCR primers' sequences for targeted genes are shown in Table S3. The sequences for GAPDH: GAPDH-F (5'-TGCACCACCAACTGCTTAGC - $\left.3^{\prime}\right)$ and GAPDH-R (5'-GGCATGGACTGTGGTCATGAG -3'). Expression value of the targeted gene in a given sample was normalized to the corresponding expression of GAPDH. The $2^{-\Delta \Delta \mathrm{Ct}}$ method was used to calculate relative expression of the targeted genes.

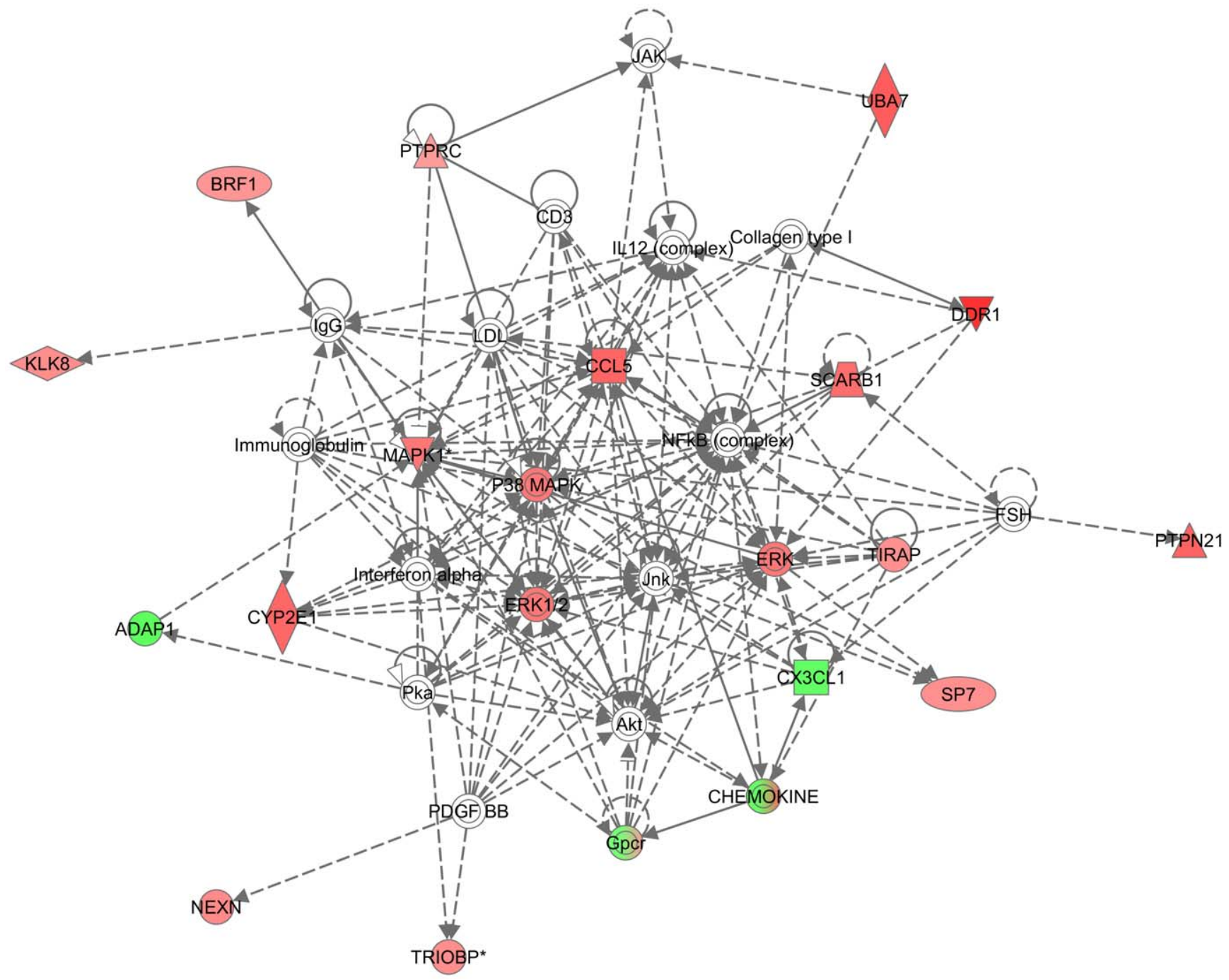

Figure 4. Top one network dysregulated in SIM2 ${ }^{\text {low }}$. This network contained 16 focus genes with a score of 29. Different shapes of the node represent different groups of the focus genes. The intensity of the node color indicated the degree of the up (red) and down (green) gene expression level. The top functions of this network are cellular movement, immune cell trafficking, organismal injury and abnormalities. doi:10.1371/journal.pone.0028837.g004 


\section{Results}

SIM2 gene is differentially expressed in prostate normal and cancer prostatectomy and cell lines

We have evaluated SIM2 gene expression in a total of 24 normal and tumor prostatectomy samples shown in table 1 . Because SIM2 gene exists in two isoforms, SIM2 short (SIM2s) and SIM2 long (SIM2L), we confirmed the expression of both isoforms in RNA extracted from prostatectomy using branched DNA technique (Fig. 1A \& 1B). SIM2s and SIM2L showed significant overexpression in tumor samples when compared to benign samples, with $\mathrm{p}<0.000003$ and $\mathrm{p}<0.00005$, respectively. However, the ratio of SIM2s to SIM2L expression was no difference between benign and tumor (table 1, T-test with $\mathrm{p}=$ 0.85). The SIM2s and SIM2L expression were 7.03 and 6.95 times higher respectively in the tumors comparing the means of the two groups after a $\log$ adjustment to assure normality and constant variance within each group. Expression of SIM2s and SIM2L was also evaluated in four human prostate cancer cell lines, PC3, LNCaP, DU145 and VCaP, and in the normal prostate epithelial cell line PrEG. Both SIM2s and SIM2L isoforms were highly expressed in $\mathrm{VCaP}$ cells, while there was a moderate expression level in PC3 cells and very low expression in DU145, LNCaP, and PrEC cells (Fig. 1C). Because there are only a few available antibodies to SIM2, we have only been able to clearly identify the short isoform of SIM2 (SIM2s) in cellular protein extracts by western blot. This scarcity of antibodies complicated our task of studying the function of SIM2 long isoform. The SIM2s protein expression level was consistent with its gene expression in prostate normal and cancer cell lines. (Fig. 1D).

\section{Silencing SIM2 expression in PC3 cells}

To achieve the highest downregulation of SIM2 expression using lentiviral shRNA, we have selected the PC3 cell line as a model. PC3 cells were transduced with five different SIM2 shRNA expression vectors, four of which (shRNA48, shRNA49, shRNA50 and shRNA51) showed significant inhibitory effect compared to control shRNAs. Over $80 \%$ silencing of gene expression was achieved using shRNA51 (Fig. 2A\&B). Two control cell lines were generated using either a vector stably expressing shRNA targeting luciferase or an empty vector. A similar inhibitory pattern was observed for SIM2L gene expression in these stably infected PC3 cell lines. Similarly, efficient transient silencing of SIM2S and SIM2L was achieved in PC3 (Figure S1).

\section{Impact of SIM2 silencing on gene expression profile in PC3 cells}

Despite its suspected role in cancer, very little is known about the contribution of the transcription factor SIM2 to the regulation
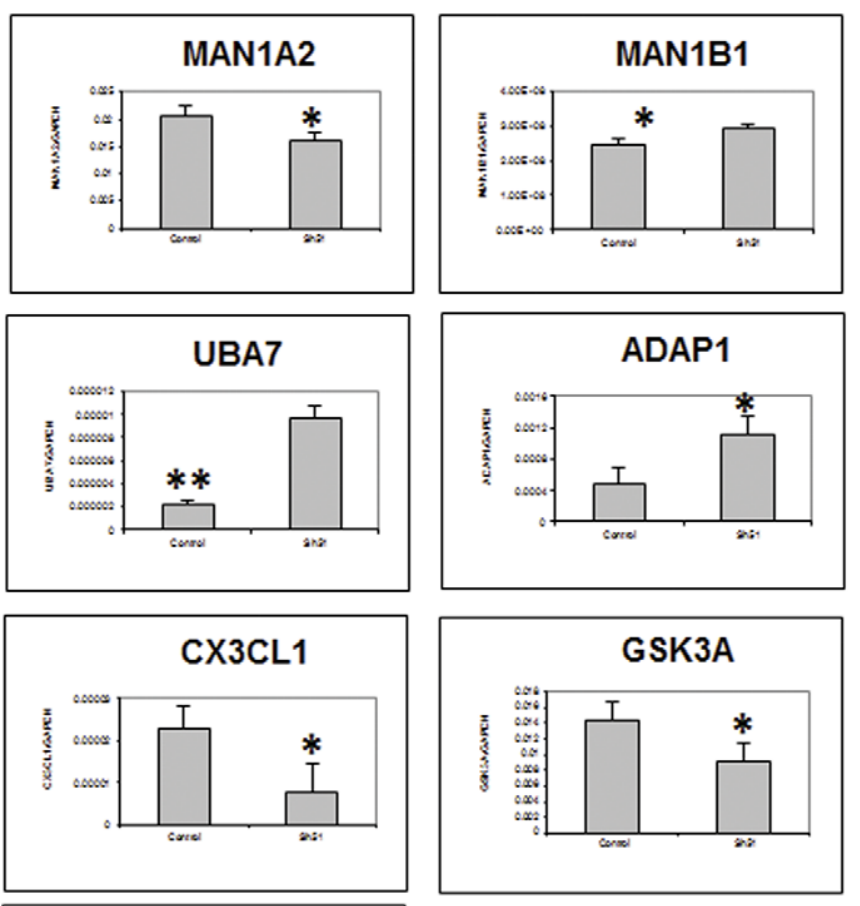
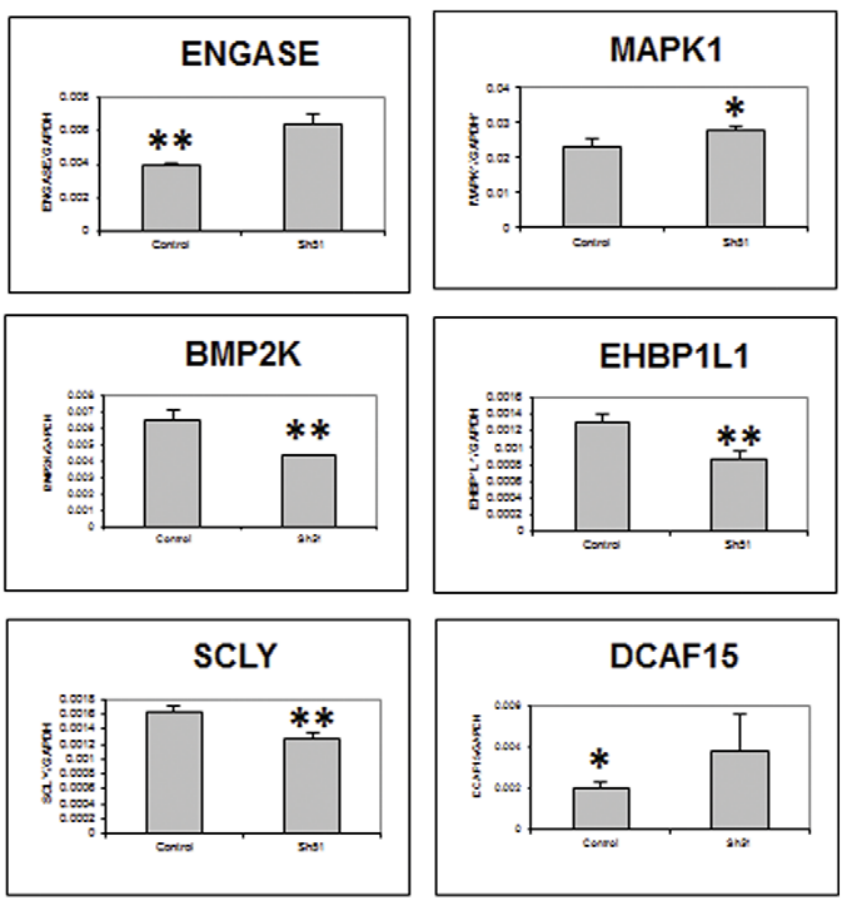

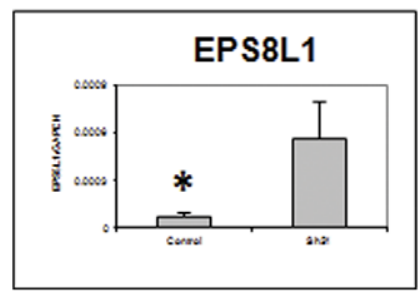

Figure 5. Validation of selected genes from stable transfectant by qRT-PCR. qRT-PCR validation of mRNA expression levels of individual genes was performed by two-step real-time RT-PCR analysis on Applied Biosystems 7900HT Prism instrument. ${ }^{*}, \mathrm{P}<.05 ;{ }^{* *}, \mathrm{P}<.01 ;{ }^{* * *}, \mathrm{P}<.001$. Measurements were performed in triplicates and data presented as Mean \pm SD.

doi:10.1371/journal.pone.0028837.g005 

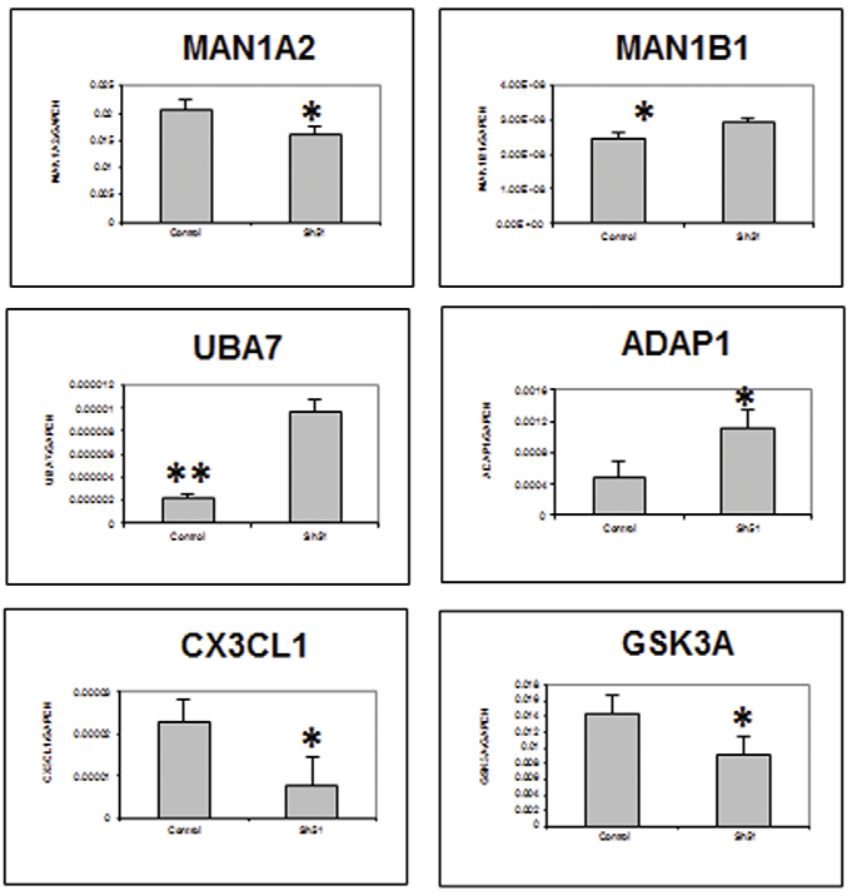
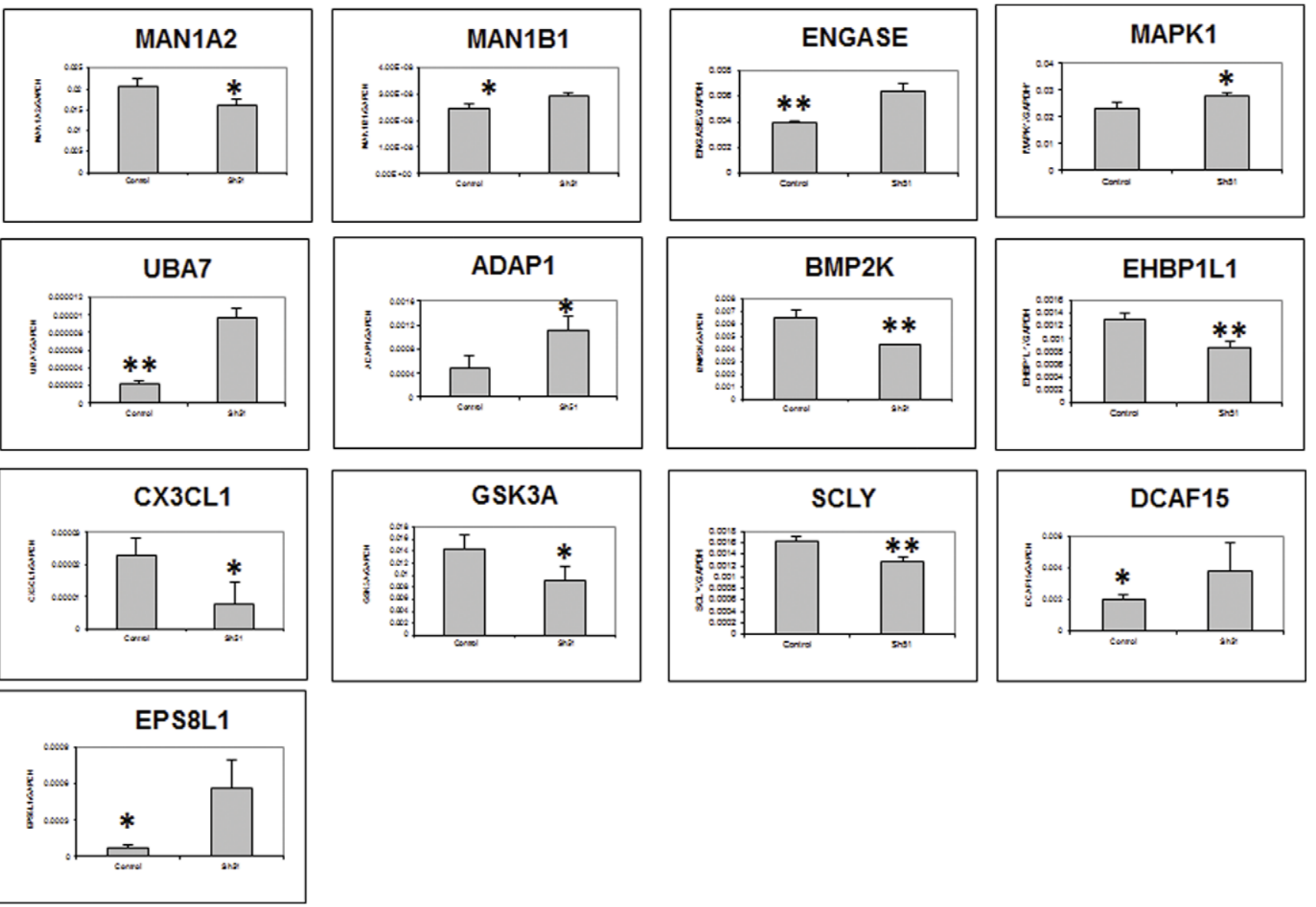

Figure 6. Validation of selected genes from transient transfectant by qRT-PCR. qRT-PCR validation of mRNA expression levels of individual genes was performed by two-step real-time RT-PCR analysis on Applied Biosystems 7900HT Prism instrument. ${ }^{*}, \mathrm{P}<.05 ;{ }^{* *}, \mathrm{P}<.01 ;{ }^{* * *}, \mathrm{P}<.001$. Measurements were performed in triplicates and data presented as Mean \pm SD.

doi:10.1371/journal.pone.0028837.g006

of gene expression [23]. We therefore examined the effects of downregulation of SIM2 in prostate cancer cells. To this end, the shRNA which yielded the highest silencing rate of SIM2, i.e. shRNA51, was selected. PC3 cells treated with shRNA51 were compared to a control shRNA (shRNAc).

Gene expression profiles of PC3 SIM2 ${ }^{\text {low }}$ and control PC3 cell lines were evaluated using Affymetrix GeneChip U133 array (Plus 2.0 chip) consisting of $>52,000$ transcripts from whole human genome transcripts. Figure 1 is a heat map showing the gene dysregulation after knocking down the expression of SIM2 in PC3 cells. The expression of a large number of transcripts exhibited a change of at least 2-fold (Figure 3A and Table S4). Pathway analysis revealed that many highly differentially expressed transcripts represent genes that belong to known signaling pathways, such as the PTEN and PI3K/AKT signaling pathways (Figure 3B), whose involvement in tumorigenesis is well documented $[24,25]$. Specific genes involved in each signaling pathway are shown in Table S1. Among those genes, CCL5, MAPK1, P38, DDR 1 and ERK played a central role in the pathway network (Figure 4). The genes in this network have been involved in cell death, metabolism, cellular development, and tumor antigen presentation. More genes involved in the highest score networks are shown in Table S2. Further analysis showed that a number of important biological functions are dysregulated following SIM2 silencing (Figure 3C). Interestingly, several cell functions related to metabolism, such as drug metabolism and metabolic disease, are among the top ranked functions.

Validation by RT-PCR of a group of differentially expressed genes (Table S3) partially confirmed our in silico analysis of stable and transient transfectant PC3 cells (Figures 5 \& 6).

Table 2. Top dysregulated metabolic pathways in Sim2 $2^{\text {low }}$ PC3 cells.

\begin{tabular}{lll}
\hline Pathway Name & $\begin{array}{l}\text { Total number of } \\
\text { Metabolites }\end{array}$ & Hits \\
\hline Purine Metabolism & 92 & 11 \\
Pyrimidine metabolism & 60 & 6 \\
Glycolysis or & 31 & 3 \\
Gluconeogenesis & 24 & 2 \\
Thiamine metabolism & 32 & 2 \\
Pyruvate metabolism & & \\
\hline
\end{tabular}

Metabolites were measured using mass spectrometry and data were analyzed using MetaboAnalyst software.

doi:10.1371/journal.pone.0028837.t002 


\section{PC3 SIM2 ${ }^{\text {low }}$ cells showed major alterations in their metabolic profile}

We sought to determine whether gene expression changes result in significant shifts in metabolic pathways in PC3 SIM2 ${ }^{\text {low }}$ cells. This was addressed by measuring 255 polar metabolites using targeted mass spectrometry (LC/MS/MS). Comparison of the metabolic profile of control cells to shRNA-SIM2-treated cells showed significant changes in several metabolic pathways and the production of 39 metabolites (Tables 2\&3). The purine metabolism pathway was the top one dysregulated pathway with 11 metabolites significantly up- or down-regulated levels out of total 92 metabolites in this pathway in SIM2 silencing PC3 cells. Pyrimidine metabolism pathway listed as the second dysregulated pathway with 6 out of total of 60 metabolites with significant changed levels (Table 2, Fig. 7). The significant alterations to the nucleic acid metabolism may indicate its important role in the prostate cancer development.

\section{Discussion}

In our previous biomarker identification efforts, we have identified SIM2 as a potential biomarker for PCa. Thanks to its overexpression in prostate tumors and its highly restricted expression in humans, we proposed to use SIM2 as an immunotherapy target and were able to identify 5 HLA-A2.1, SIM2-derived immunogenic epitopes [12]. In the present study we attempted to characterize the role of SIM2 in prostate cancer using a short hairpin RNA-induced gene silencing approach in PC3 cells as a model. We focused on profiling both the transcriptome and metabolome in SIM $2^{\text {low }}$ and normal PC3 cells, and evaluated the impact of SIM2 silencing on cell signaling and function.

The SIM2s isoform has been reported to be expressed in colon, pancreas, and prostate tumors while absent in the corresponding benign tissues [8]. We found that SIM2 genes are detectable in all these prostate cancer cells by real time PCR. However the expression levels in DU145 and LNCaP are relatively lower than other prostate cancer cells while PC3 cells express moderate level of SIM2 genes which are consistent with other report [14].

The whole spectrum of regulation of gene expression by the transcription factor SIM2 is still poorly defined. The level of regulation could be reflected by the differential expression of about 200 genes as revealed through gene expression profiling of PC3 SIM2 ${ }^{\text {low }}$ cells. Other groups have reported specific genes that are regulated by SIM2. The bHLH/PAS transcription factor single minded $2 \mathrm{~s}$ was reported to promote mammary gland lactogenic differentiation by regulation of Csn2 expression [26]. SIM2 regulates the expression of MMP-2 and TIMP-2, which drive its role in glioblastoma cells [11]. SIM2s represses BNIP3, a proapoptotic gene, through its hypoxic response element in PC3 cells [14]. Our gene expression profile in PC3 SIM2 ${ }^{\text {low }}$ cells showed significant change in PTEN, PI3K/AKT and Toll-like receptor (TLR) signaling pathways which are involved largely in the tumor progression. PTEN negatively controls the PI3K signaling pathway for cell growth and survival by dephosphorylating the 3 position of phosphoinositides [24,25]. TLR regulates cell proliferation and survival and central signaling molecules mitogenactivated protein kinase (MAPK) and PI3K play key roles [27]. Our data show that inhibition of Sim2 gene in PC3 cells affects expression of several genes encoding proteins that are organized in a network around p38MAPK. These proteins, which include CCL5, MAPKs, ERK and DDR1 (Figure 4), have been reported to be involved in tumor development. The chemokine CCL5 has been reported to be expressed by prostate cells and affect their
Table 3. List of dysregulated metabolites in Sim $2^{\text {low }}$ PC3 cells.

\begin{tabular}{|c|c|}
\hline Metabolite & P value* \\
\hline UTP & 0.00021736 \\
\hline CTP & 0.000266674 \\
\hline Thiamine-phosphate & 0.00027101 \\
\hline CDP & 0.00027427 \\
\hline ATP & 0.00032043 \\
\hline GDP & 0.00047402 \\
\hline 2-ketohaxanoic acid & 0.00099085 \\
\hline dGTP & 0.0011366 \\
\hline Adenosine & 0.0025014 \\
\hline allantoate & 0.0031712 \\
\hline 4-Pyridoxic acid & 0.0032341 \\
\hline Hydroxyphenylacetic acid & 0.004154 \\
\hline IMP & 0.0046357 \\
\hline Inosine & 0.0052766 \\
\hline xanthosine & 0.0056235 \\
\hline N-carbamoyl-L-aspartate & 0.0057818 \\
\hline CDP-choline & 0.0067616 \\
\hline lactate & 0.007935 \\
\hline GTP & 0.0084853 \\
\hline dCTP & 0.011521 \\
\hline dATP & 0.011737 \\
\hline Geranyl-PP & 0.014616 \\
\hline Thiamine pyrophosphate & 0.017058 \\
\hline dihydroxy-acetone-phosphate & 0.017355 \\
\hline AMP & 0.018114 \\
\hline Sn-glycerol-3-phosphate & 0.019883 \\
\hline 3-phosphoglycerate & 0.021186 \\
\hline UDP & 0.023169 \\
\hline dAMP & 0.023309 \\
\hline Guanine & 0.025068 \\
\hline Glucose-6-phosphate & 0.027664 \\
\hline Fructose-6-phosphate & 0.035608 \\
\hline Hexose-phosphate & 0.036721 \\
\hline dTMP & 0.044375 \\
\hline Guanosine 5-diphosphate, 3-diphosphate & 0.046342 \\
\hline 2-Isopropylmalic acid & 0.047328 \\
\hline GMP & 0.04827 \\
\hline hydroxyproline & 0.049448 \\
\hline
\end{tabular}

Metabolites were quantitated using mass spectrometry and data were analyzed using MetaboAnalyst software.

${ }^{*} \mathrm{P}<0.05$.

doi:10.1371/journal.pone.0028837.t003

growth and survival. Following activation of MAPKs p38 and ERK1/2 in LNCaP cells, the expression of CCL5 increases, resulting in enhanced cell proliferation [28,29]. PC3 cell proliferation and invasion were also significantly suppressed after DDR1 knockdown by siRNA [30,31].

Our RT-PGR data revealed discrepancies between transient and stable silencing of SIM2 in PC3 cells. This may be a result of 1) the presence of two isoforms of SIM2 that are silenced to 


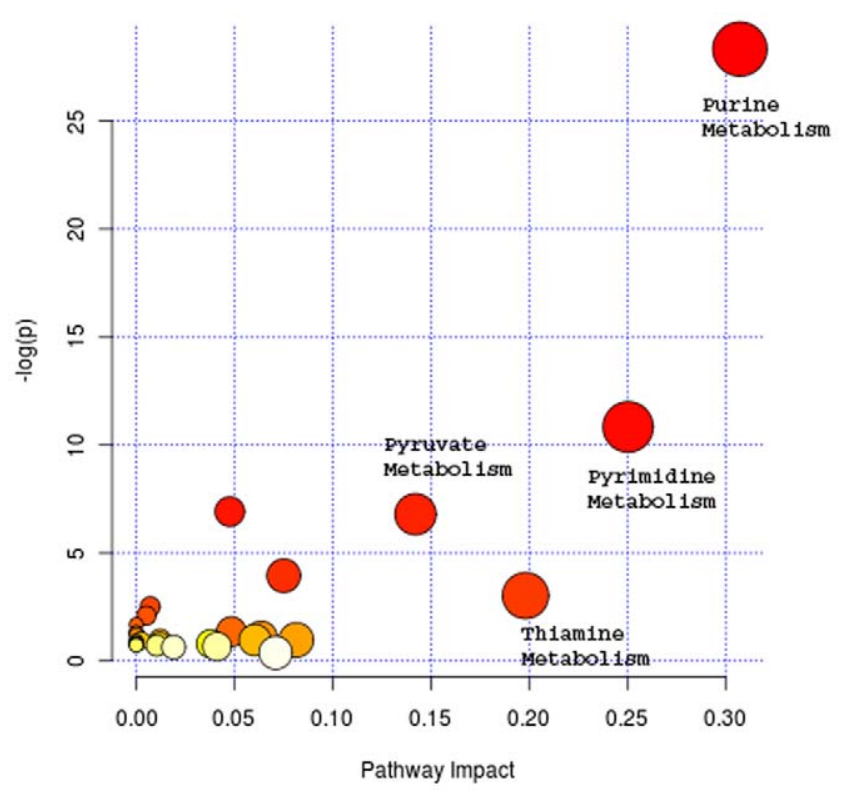

Figure 7. PC3 SIM2-shRNA cells showed major alterations in purine and pyrimidine metabolism pathways. Metabolites were extracted from SIM2 ${ }^{\text {low }}$ and normal PC3 cells using methanol and the abundance of 239 metabolites were measured using targeted LC/MS/ MS. Data were analyzed using MetaboAnalyst software. The metabolic pathways arranged according to the scores from enrichment analysis ( $y$ axis) and from topology analysis ( $\mathrm{x}$ axis). Triplicate measurements were performed.

doi:10.1371/journal.pone.0028837.g007

different extents in both setups, or 2) SIM2 may regulate gene expression of other genes either directly or indirectly.

Function analysis also revealed that three functions related to cell metabolism had been dysregulated in the PC3 SIM2 ${ }^{\text {low }}$ cells. This suggested that SIM2 might have metabolic consequences. We have evaluated the production by PC3 cells of 255 metabolites that encompass a large number of human metabolic pathways. Of these, data were obtained for 239 metabolites. Our analysis revealed significant changes in metabolites that constitute key pathways, such as the purine and pyrimidine pathways.

Suppression of SIM2 short isoform (SIM2s) by antisense oligonucleotides reduced tumor growth in colon cancer cells and induced CAPAN-1 pancreatic cell death through apoptosis $[7,8,9]$. SIM2s was also reported to be an aggressive prostate cancer biomarker since SIM2s protein was associated with increased preoperative serum prostate specific antigen (PSA), high histological grade, invasive tumor growth and increased tumor cell

\section{References}

1. Chrast R, Scott HS, Chen H, Kudoh J, Rossier C, et al. (1997) Cloning of two human homologs of the Drosophila single-minded gene SIM1 on chromosome $6 q$ and SIM2 on 21q within the Down syndrome chromosomal region. Genome Res 7: 615-624.

2. Moffett P, Reece M, Pelletier J (1997) The murine Sim-2 gene product inhibits transcription by active repression and functional interference. Mol Cell Biol 17: 4933-4947.

3. Ema M, Ikegami S, Hosoya T, Mimura J, Ohtani H, et al. (1999) Mild impairment of learning and memory in mice overexpressing the mSim2 gene located on chromosome 16: an animal model of Down's syndrome. Hum Mol Genet 8: 1409-1415.

4. Moffett P, Pelletier J (2000) Different transcriptional properties of mSim-1 and mSim-2. FEBS Lett 466: 80-86.

5. Yamaki A, Tochigi J, Kudoh J, Minoshima S, Shimizu N, et al. (2001) Molecular mechanisms of human single-minded 2 (SIM2) gene expression: identification of a promoter site in the SIM2 genomic sequence. Gene 270: 265-275. proliferation [13]. A recent study showed that SIIM2s may attenuate cell death processes through BNIP3 repression in PC3AR+ cells. However, knockdown of SIM2s in breast cancer MCF-7 cells increased tumorigenesis and thus showed tumor suppressor activity $[32,33]$. Most of the previous studies focused on the SIM2s by either intruding or knockdown of SIM2s, we are lacking of the data clarifying the functional role of SIM2 protein including both of its isoforms. Our study reported a combined role of both isoforms of the SIM2 implicated in the prostate cancer cell. Distinguishing the roles of SIM2s and SIM2L may have more profound meaning to understand the functional role of SIM2 in prostate cancer progression, which is our next step to uncover more significance of this gene.

\section{Supporting Information}

Figure S1 Transient silencing of SIM2s and SIM2L expression in PC3 cells. PC3 cells were transduced with either a control (Ctrl) or shRNA51 (sh51) and cultured in the presence of puromycin for 3 days. Real time RT-PCR was performed in triplicates to evaluate gene expression of SIM2 s (Upper Panel) and SIM2L (Lower Panel).

(TIF)

Table S1 The top Dysregulated Signaling Pathways in SIM2 ${ }^{\text {low }}$ cells. Top dysregulated canonical pathways were identified through analysis of differentially expressed gene data, using Ingenuity Pathway Analysis package.

(DOC)

Table S2 The Molecules in the Highest Score Networks in SIM2 ${ }^{\text {low }}$ cells. Data representing differentially expressed genes were submitted to Ingenuity Pathway Analysis package and high score networks were identified.

(DOG)

Table S3 List of primers used for RT-PCR quantitation of expression of of selected genes. The primers were designed using Pimer3 program: http://frodo.wi.mit.edu/primer3/.

(DOC)

Table S4 Gene expressions that were either up- or downregulated greater than 2 fold in the SIM2 $2^{\text {low }}$.

(XLSX)

\section{Author Contributions}

Conceived and designed the experiments: BL JMA MGS MSA. Performed the experiments: BL JMA MSA. Analyzed the data: BL JMA MGS MSA. Contributed reagents/materials/analysis tools: MSA JMA. Wrote the paper: BL MGS MSA.

6. Deyoung MP, Scheurle D, Damania H, Zylberberg C, Narayanan R (2002) Down's syndrome-associated single minded gene as a novel tumor marker. Anticancer Res 22: 3149-3157.

7. DeYoung MP, Tress M, Narayanan R (2003) Identification of Down's syndrome critical locus gene SIM2-s as a drug therapy target for solid tumors. Proc Natl Acad Sci U S A 100: 4760-4765.

8. DeYoung MP, Tress M, Narayanan R (2003) Down's syndrome-associated Single Minded 2 gene as a pancreatic cancer drug therapy target. Cancer Lett 200: 25-31.

9. Aleman MJ, DeYoung MP, Tress M, Keating P, Perry GW, et al. (2005) Inhibition of Single Minded 2 gene expression mediates tumor-selective apoptosis and differentiation in human colon cancer cells. Proc Natl Acad Sci U S A 102: 12765-12770.

10. Kwak HI, Gustafson T, Metz RP, Laffin B, Schedin P, et al. (2007) Inhibition of breast cancer growth and invasion by single-minded 2s. Carcinogenesis 28: 259-266. 
11. He Q, Li G, Su Y, Shen J, Liu QO Single minded 2-s (SIM2-s) gene is expressed in human GBM cells and involved in GBM invasion. Cancer Biol Ther 9: 430-436.

12. Arredouani MS, Lu B, Bhasin M, Eljanne M, Yue W, et al. (2009) Identification of the transcription factor single-minded homologue 2 as a potential biomarker and immunotherapy target in prostate cancer. Clin Cancer Res 15: 5794-5802.

13. Halvorsen OJ, Rostad K, Oyan AM, Puntervoll H, Bo TH, et al. (2007) Increased expression of SIM2-s protein is a novel marker of aggressive prostate cancer. Clin Cancer Res 13: 892-897.

14. Farrall AL, Whitelaw ML (2009) The HIFlalpha-inducible pro-cell death gene BNIP3 is a novel target of SIM2s repression through cross-talk on the hypoxia response element. Oncogene 28: 3671-3680.

15. Jones J, Otu H, Spentzos D, Kolia S, Inan M, et al. (2005) Gene signatures of progression and metastasis in renal cell cancer. Clin Cancer Res 11: 5730-5739.

16. Li C, Wong WH (2001) Model-based analysis of oligonucleotide arrays: expression index computation and outlier detection. Proc Natl Acad Sci U S A 98: 31-36.

17. Yuen T, Wurmbach E, Pfeffer RL, Ebersole BJ, Sealfon SC (2002) Accuracy and calibration of commercial oligonucleotide and custom cDNA microarrays. Nucleic Acids Res 30: e48.

18. Ramalho-Santos M, Yoon S, Matsuzaki Y, Mulligan RG, Melton DA (2002) "Stemness": transcriptional profiling of embryonic and adult stem cells. Science 298: 597-600.

19. Haram KM, Peltier HJ, Lu B, Bhasin M, Otu HH, et al. (2008) Gene expression profile of mouse prostate tumors reveals dysregulations in major biological processes and identifies potential murine targets for preclinical development of human prostate cancer therapy. Prostate 68: 1517-1530.

20. Livak KJ, Schmittgen TD (2001) Analysis of relative gene expression data using real-time quantitative PCR and the 2(-Delta Delta C(T)) Method. Methods 25: 402-408.

21. Xia J, Psychogios N, Young N, Wishart DS (2009) MetaboAnalyst: a web server for metabolomic data analysis and interpretation. Nucleic Acids Res 37: W652-660.
22. Xia J, Wishart DS Web-based inference of biological patterns, functions and pathways from metabolomic data using MetaboAnalyst. Nat Protoc 6: 743-760.

23. Metz RP, Kwak HI, Gustafson T, Laffin B, Porter WW (2006) Differential transcriptional regulation by mouse single-minded 2s. J Biol Chem 281: 10839-10848.

24. Cantley LC, Neel BG (1999) New insights into tumor suppression: PTEN suppresses tumor formation by restraining the phosphoinositide 3-kinase/AKT pathway. Proc Natl Acad Sci U S A 96: 4240-4245.

25. Yuan TL, Cantley LC (2008) PI3K pathway alterations in cancer: variations on a theme. Oncogene 27: 5497-5510.

26. Wellberg E, Metz RP, Parker C, Porter WW The bHLH/PAS transcription factor singleminded $2 \mathrm{~s}$ promotes mammary gland lactogenic differentiation. Development 137: 945-952.

27. Li X, Jiang S, Tapping RI Toll-like receptor signaling in cell proliferation and survival. Cytokine 49: 1-9.

28. Colombatti M, Grasso S, Porzia A, Fracasso G, Scupoli MT, et al. (2009) The prostate specific membrane antigen regulates the expression of IL-6 and CCL5 in prostate tumour cells by activating the MAPK pathways. PLoS One 4: e4608.

29. Vaday GG, Peehl DM, Kadam PA, Lawrence DM (2006) Expression of CGL5 (RANTES) and CCR5 in prostate cancer. Prostate 66: 124-134.

30. Shimada K, Nakamura M, Ishida E, Higuchi T, Yamamoto H, et al. (2008) Prostate cancer antigen-1 contributes to cell survival and invasion though discoidin receptor 1 in human prostate cancer. Cancer Sci 99: 39-45.

31. Yamanaka R, Arao T, Yajima N, Tsuchiya N, Homma J, et al. (2006) Identification of expressed genes characterizing long-term survival in malignant glioma patients. Oncogene 25: 5994-6002.

32. Gustafson TL, Wellberg E, Laffin B, Schilling L, Metz RP, et al. (2009) Ha-Ras transformation of MCF10A cells leads to repression of Singleminded-2s through NOTCH and C/EBPbeta. Oncogene 28: 1561-1568.

33. Laffin B, Wellberg E, Kwak HI, Burghardt RC, Metz RP, et al. (2008) Loss of singleminded-2s in the mouse mammary gland induces an epithelial-mesenchymal transition associated with up-regulation of slug and matrix metalloprotease 2. Mol Cell Biol 28: 1936-1946. 